\title{
Comparación de la productividad primaria con aplicaciones de lombriabono y fertilizante comercial, en cultivos de camarones blancos (Litopenaeus vannamei)
}

\section{Comparison of primary productivity with worm fertilizer and commercial fertilizer applications in white shrimp (Litopenaeus vannamei)}

\author{
Isamar Martínez ${ }^{1}$ \\ Anielka Amaya ${ }^{2}$ \\ César Arrieta ${ }^{3}$ \\ Martha Roque 4 \\ Carmen Hernández ${ }^{5}$ \\ Noelia Cea ${ }^{6}$ \\ Brenda Torres ${ }^{7}$
}

\section{Resumen}

El fitoplancton es el primer eslabón en la cadena alimenticia de todo organismo acuático, constituye fertilización indispensable, como promotor de la productividad primaria, y el uso de fertilizantes se convierte en el segundo insumo de mayor costo en la producción acuícola, por esta razón nace la urgencia de búsqueda de fertilizaciones alternas que reduzcan el costo de este insumo y por ende, el costo total de la producción, esto conllevo a la realización del ensayo utilizando el Lombriabono (extracto sólido del humus de la lombriz Eisenia foetida), como una alternativa orgánica y amigable con el ambiente. Este estudio se realizó en el Laboratorio de Investigaciones Marinas y Acuícolas (LIMA) de la UNAN- León, ubicado a $22 \mathrm{~km}$ del municipio de León (comunidad Las Peñitas). El análisis estadístico se hizo a través de la prueba de normalidad de Kolmogorov Smirnov. El propósito fue evaluar su efecto promotor en el fitoplancton en contraste con los efectos del fertilizante comercial, aplicando dosis de 10olb/Ha. de cada fertilizante para cada tratamiento, siendo el T1: Fertilizante comercial y el T2: Fertilizante de Lombriabono, durante el período de experimentación, logrando resultados de variables físico-químicas (oxígeno, $\mathrm{pH}$, temperatura, salinidad y turbidez) para ambos tratamientos sin variaciones relevantes, con la presencia de

1 Ingeniera Acuícola. Email: isamartinez19@yahoo.com ORCID https://orcid.org/oooo-00o2-4282-6885

2 Ingeniera Acuícola. Email: amayaanielka@yahoo.com ORCID https://orcid.org/oooo-0002-4211-9929

3 Ingeniero Acuícola. Email: arrietace@yahoo.com ORCID https://orcid.org/oooo-0oo2-1689-5895

4 Docente del Departamento de Acuícola de la UNAN-León. Email: martha.roque@ev.unanleon.edu.ni ORCID https://orcid. org/oooo-0002-9054-9640

5 Master en desarrollo local sostenible. Jefa de Departamento de Acuícola de la UNAN-León. Email: carmen.hernandez@ev.unanleon. edu.ni; No. ORCID https://orcid.org/oooo-00o2-3002-5255

6 Docente del Departamento de Acuícola de la UNAN-León. Email: noelia.cea@ev.unanleon.edu.ni ORCID: https://orcid. org/oooo-0oo1-7913-9459

7 Docente del Departamento de Acuícola de la UNAN-León. Email: Brenda.torres@ev.unanleon.edu.ni ORCID: https://orcid.org/oooo-ooo2-1441-2386

Recibido: 04/06/2019 Aprobado: 19/06/2019

Martínez, I., Amaya, A., Arrieta, C., Roque, M., Hernández, C., Cea, N., \& Torres, B. (2019). Comparación de la productividad primaria con aplicaciones de lombriabono y fertilizante comercial, en cultivos de camarones blancos (Litopenaeus vannamei). Ciencia E Interculturalidad, 24(01), 174-188. https://doi.org/10.5377/rci.v24i01.8013 
los grupos de Bacillariophyta y Chlorophyta en mayor cantidad y Cianophyta en menor cantidad, así mismo se encontraron especies como C. meduanae, C. atomus, N. tripunctata, Nitzschia reversa, Cosmarium Contractum, Volvox aureus, D. tertiolecta.

Palabras clave: Cultivo; Camarón; Fertilizante; Microalgas; Productividad.

\section{Abstract}

The phytoplankton is the first link in the food chain of any aquatic organism, it constitutes indispensable fertilization, as a promoter of primary productivity, and the use of fertilizers becomes the second most expensive input in aquaculture production, for this reason the urgency to search for alternate fertilizations that reduce the cost of this input and, therefore, the total production cost, this led to the trial using the worm fertilizer (solid extract of the earthworm Eisenia foetida), as an organic alternative and friendly with the environment. This study was conducted at the Marine and Aquaculture Research Laboratory (LIMA) of UNAN-León, located $22 \mathrm{~km}$ from the municipality of León (Las Peñitas Community). The statistical analysis was done through the Kolmogorov Smirnov normality test. The purpose was to evaluate its promoter effect in phytoplankton in contrast to the effects of commercial fertilizer, applying doses of $100 \mathrm{lb} / \mathrm{Ha}$. of each fertilizer for each treatment, being T1: commercial Fertilizer and T2: The worm fertilizer, during the period of experimentation, obtaining results of physical-chemical variables (oxygen, $\mathrm{pH}$, temperature, salinity and turbidity) for both treatments without relevant variations, with the presence of the Bacillariophyta and Chlorophyta groups in greater quantity and Cianophyta in smaller quantity, likewise species such as C. meduanae, C. atomus, N. tripunctata, Reverse Nitzschia, Cosmarium Contractum, Volvox aureus, D. tertiolecta.

Keywords: Culture; Shrimp; Fertilizer; Microalgae; Productivity.

\section{Introducción}

La acuicultura es considerada como el cultivo de especies acuáticas, ya sean microalgas, peces, moluscos y crustáceos. En estos últimos años, el organismo de mayor relevancia para la acuicultura ha sido el camarón blanco del Pacífico Litopenaeus vannamei, representando hoy casi el $65 \%$ de la producción mundial y se prevé que esta tendencia se mantenga (FAO, 2016), en Nicaragua el sector ha experimentado un incremento significativo en los últimos años, caracterizado por una tasa de crecimiento anual del $9 \%$ al 10\%, ocasionando un creciente interés en el desarrollo del cultivo de camarón, pero en busca de alternativas que permitan reducir los costos de producción.

Una opción económica viable para reducir estos costos en la fertilización es la utilización de abonos orgánicos, algunos de éstos son productos de origen agropecuario que se utilizan para el mejoramiento de suelos agrícolas destinados al cultivo de 
granos básicos (arroz, frijoles, maíz, etc.), o bien para cultivo de hortalizas. Cuando el fertilizante orgánico es agregado a las piscinas es descompuesto por las bacterias con lo cual se liberan los nutrientes requeridos para el crecimiento del fitoplancton. Entre los abonos orgánicos cabe destacar el humus de lombriz, que se obtiene mediante el proceso de la lombricultura; una biotecnología que utiliza la lombriz roja Eisenia foetida, como un transformador natural que recicla todo tipo de materia orgánica (Guerra, 2011).

\section{Revisión Literaria}

En Nicaragua la camaronicultura ha venido creciendo exponencialmente y con ella, el valor adquisitivo de insumos como alimento y fertilizante y abarca el 50\% del costo total de la producción, razón por la cual se ha generado una búsqueda constante de alternativas que minimicen los costos de los insumos más importantes en la producción, por lo que en este estudio se propone una alternativa de fertilizante orgánico que disminuya el costo de compra de este producto basándonos en estudios previos a este, tales como el realizado por Bhusan y Yadav (2003), afirman que el humus contiene una mayor cantidad de carbono y fósforo, menos potasio y nitrógeno que aquella materia orgánica no transformada; además es rico en nutrientes, vitaminas enzimas, antibióticos y promotores de crecimiento, recientemente, Ruíz (2011), reafirma las propiedades del humus de lombriz como fertilizante orgánico, aporta además de lo mencionado el elevado contenido de ácidos húmicos, fúlvicos y producción de hormonas como el ácido indolacético y giberélico que estimulan el crecimiento y funciones vitales de las diatomeas. Ruiz, (2013), implementa el uso de fertilizante a base de humus de lombriz en cultivo de camarones teniendo mayores cantidades de fitoplancton a diferencia del resto de los tratamientos implementados como urea, además de presentar una adecuada calidad de agua para el crecimiento del camarón blanco, por lo que recomienda la implementación de este tipo de fertilizante en las actividades camaronicolas, beneficiando la reducción de gastos en fertilización y alimentación los cuales superan más de 50\% de los costos operativos de esta actividad.

Es realmente poca la información que existe sobre el uso de humus de lombriz como fertilizante de lombriabono en estanques para camarón Litopenaeus vannamei y la existente es de su uso en la fertilización en estanques de piscicultura. Ya que la utilización de lombriabono en piscicultura ha tenido beneficios en la producción de fitoplancton, en el presente estudio se evalúa la aplicación del humus de lombriz en la productividad de fitoplancton y en el crecimiento de camarón en estanques.

\section{Materiales y métodos}

Este estudio se llevó a cabo en el Laboratorio de Investigaciones Marinas y Acuícolas (LIMA) de la UNAN- León, ubicado a 22 km del municipio de León (comunidad Las Peñitas). 
El análisis estadístico se hizo a través de la prueba de normalidad de Kolmogorov Smirnov. El ensayo contó con 2 recipientes plásticos con capacidad de 1 tonelada, el agua utilizada fue bombeada a los dos dispositivos experimentales mediante una bomba sumergible MODV SUMP PUMP modelo M1oos/m serie SR\#100894, 1.3 HP ubicada en el reservorio a través una red de tuberías de 2 pulgadas de diámetro. Cada recipiente se correspondía a un tratamiento el T1: fertilizante comercial, y T2: fertilizante Lombriabono, bajo un sistema semi-intensivo con 12 camarones/m2.

Para ambas condiciones experimentales se fertilizo a razón de 100 lbs/ha, homogenizando el fertilizante con agua hasta lograr una disolución completa y posteriormente se aplicó, con la diferencia que el fertilizante orgánico antes de su aplicación se dejó sedimentar por espacio de 1 hora. La frecuencia fue semanal, en todo el periodo que duró el estudio.

Una vez que las aguas estaban fértiles se procedió a aclimatar a las postlarvas de acuerdo a la temperatura y salinidad en que provienen, luego se realizó la siembra de estas colocándolas en los recipientes experimentales. El conteo e identificación se realizó semanalmente, para el conteo e identificación de fitoplancton se colecto en un frasco de $80 \mathrm{ml}$; inmediatamente, con una pipeta se le agrego 3 gotas de solución lugol por cada $80 \mathrm{ml}$ de la muestra.

Una vez extraída la muestra se procedió al montaje en la cámara de Neubauer, se colocó en el microscopio para observar, primero se utilizó el lente de menor aumento $(4 \mathrm{X})$ hasta obtener los cuadrantes completos, luego se observó con el lente de (10X) hasta obtener los primero 16 cuadrantes de la primera esquina, y por último se observó con el lente de (40X) para contar cada cuadrante de los primeros 16 de dicha esquina en forma de $S$ de arriba hacia abajo, en el cual se cuantifico el volumen de células/ $\mathrm{ml}$ contenida en la muestra, que fue obtenida a través de la multiplicación por 2,500 (este es de la división de factor de conversión que es 104 que representa los cuadrantes contados) por el número de microalgas encontradas de cada grupo.

Posteriormente se identificarón los diferentes grupos y especie de microalgas, a través de la cámara de Sedgwick- Rafter (S/R) de la siguiente manera: se colocó $1 \mathrm{ml}$ de la muestra girando la parte posterior del cubre objeto de tal forma que no quedaran gotas de agua. Se examinó a una magnificación de 1ox con un microscopio óptico convencional, se tomaron un total de 18 cuadrantes de la cámara, el conteo se hizo en forma de $S$, se contó el número de organismos encontrados en cada cuadrante y una vez identificados se anotó en el formato correspondiente a la cámara (S/R). Se tomó en cuenta no contar los organismos que se encontraban en los bordes, por lo que al sedimentarse la mayoría de los organismos quedan en el extremo de la cámara. Las lecturas se realizaron por tres repeticiones de cada muestra para tener un menor rango de error. 
Para medir el oxígeno disuelto y temperatura, se utilizó el oxigenómetro marca: YSI 55 y su unidad de medida es mg/L (miligramos por litros), en el caso del pH se midió a través de un pHmetro marca HANNA HI 98129, para el caso de la salinidad se manipulo un Refractómetro manual óptico (Salinómetro), Modelo: TI- RSATo10oA MARCA: TRANS INSTRUMENTS siendo su unidad de medida \%o (Partes por mil), todos los datos obtenidos se registraron en un formato de campo, las horas de monitoreo fueron a las 06:00 am y 06:00 am.

Para medir el nivel de turbidez del cuerpo de agua se utilizó un disco de Secchi. Este se sumergió (preferentemente al mediodía, ya que a esta hora del día el sol se encuentra en el punto más alto y no habrá interferencia de las nubes) a la sombra, es decir con el sol detrás del operador y en posición horizontal, mediante una cuerda graduada hasta que deja de ser visible (se anota el dato en centímetros de la profundidad hasta donde se deja de divisar el disco). Luego se asciende lentamente hasta que vuelve a ser visible (se anota el dato en centímetros de la profundidad donde es visible nuevamente el disco). El promedio entre ambas profundidades se reporta como transparencia del Disco de Secchi.

Los datos del ensayo se registraron en una bitácora, en ella, se incluyó, las observaciones, ideas, datos, de las acciones que se llevaron a cabo para el desarrollo del ensayo, teniendo un orden cronológico de acuerdo al avance del estudio. El siguiente paso en el proceso es la introducción de datos, se procedió a convertir la información recopilada a un formato electrónico de computador en el paquete SPSS versión 23, con el objetivo de revisar el supuesto de normalidad de los datos con la prueba de Kolmogorov - Smirnov, antes de aplicar la prueba $\mathrm{T}$ - Student para muestras independientes o la U de Mann Whitney, para representar los resultados a través de gráficas lineales el valor de los parámetros físicos y químicos.

Los datos de conteos de géneros presentes fueron agrupados por tratamientos y campañas de muestreo ingresándolos a planillas de cálculo y generando gráficas con el software Excel, en función de los datos ingresados se estimó la abundancia total, la riqueza de especies y se calculó con los siguientes índices ecológicos: índice de Shannon e índice de Simpson.

\section{Resultados y discusión}

\section{Oxígeno Disuelto}

El análisis estadístico a través de la prueba de Kolmogorov-Smirnov o prueba de normalidad, indican que el comportamiento de los datos no se da de forma normal al presentar un nivel de significancia menor de 0.05 , debido a que son fluctuaciones de factores ambientales. 
Reafirmando lo anterior, los resultados de Oxígeno disuelto durante el periodo de estudio para ambos tratamientos $\mathrm{T}_{1}$ (Fertilizante Comercial) y T2 (Fertilizante Lombriabono), presentan una leve tendencia a variar sus fluctuaciones en el tiempo, obteniendo datos mínimos de $0.31 \mathrm{mg} / \mathrm{Lt}$ para horas de la mañana y un máximo de $11.42 \mathrm{mg} / \mathrm{Lt}$ para horas de la tarde en el $\mathrm{T} 1$, en el caso del T2 el valor mínimo de Oxigeno fue $0.61 \mathrm{mg} / \mathrm{Lt}$ para horas de la mañana y por la tarde para el mismo tratamiento tuvimos un máximo de $15.62 \mathrm{mg} / \mathrm{Lt}$. Esta variación del oxígeno se debió principalmente al valor de $\mathrm{pH}$ presente en ambos tratamientos durante el estudio, ya que al tener $\mathrm{pH}$ bajos se da una inhibición del florecimiento algal, sumado las variaciones climáticas mencionadas anteriormente, sin dejar a un lado que en el estudio contábamos con organismos vivos que como sabemos son la principal fuente de consumo de oxígeno en un estanque acuícola, ya que al aumentar la biomasa del animal aumenta la demanda de oxigeno por parte de este y por ende los niveles del gas van disminuyendo paulatinamente en el estanque.

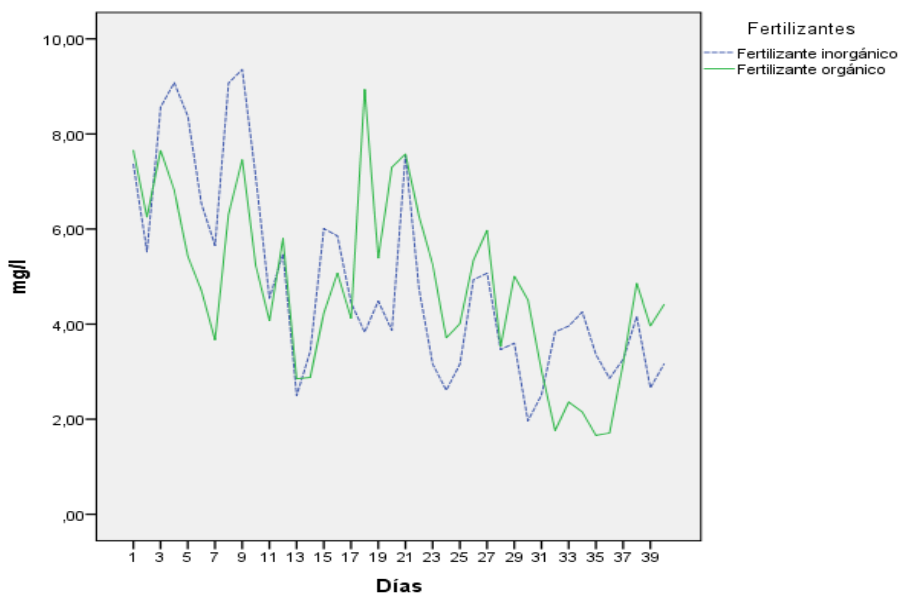

Figura No. 1: Comportamiento del Oxígeno Disuelto en cultivo de camarón, utilizando fertilizante a base de Lombriabono versus fertilizante comercial.

Los valores de $\mathrm{pH}$ durante el periodo de estudio para ambos tratamientos $\mathrm{T}_{1}$ (Fertilizante comercial) y T2 (Fertilizante Lombriabono), presentan una leve tendencia a variar sus fluctuaciones en el tiempo, obteniendo datos mínimos de 4.71 para las horas de la mañana y un máximo de 8.42 para horas de la tarde en la $\mathrm{T} 1$, en el caso de $\mathrm{T} 2$ el valor mínimo de $\mathrm{pH}$ fue 4.72 para horas de la mañana y por la tarde tuvimos un máximo 9.41. Según Benavente et al (2012) afirma que el rango de la mayoría de los cultivos de microalgas están entre 7 y 9 , en cuanto a las variaciones de $\mathrm{pH}$ que se encontraron en ambos tratamientos durante el estudio, obtuvimos descensos de $\mathrm{pH}$, esto se debió a que en las primeras semanas habían menores concentraciones de microalgas en el cuerpo de agua provocando acumulación de dióxido de carbono producto de la respiración en el estanque, ya que sin la presencia de fitoplancton no hay consumo de este gas para la producción de oxígeno a través de la actividad fotosintética. 


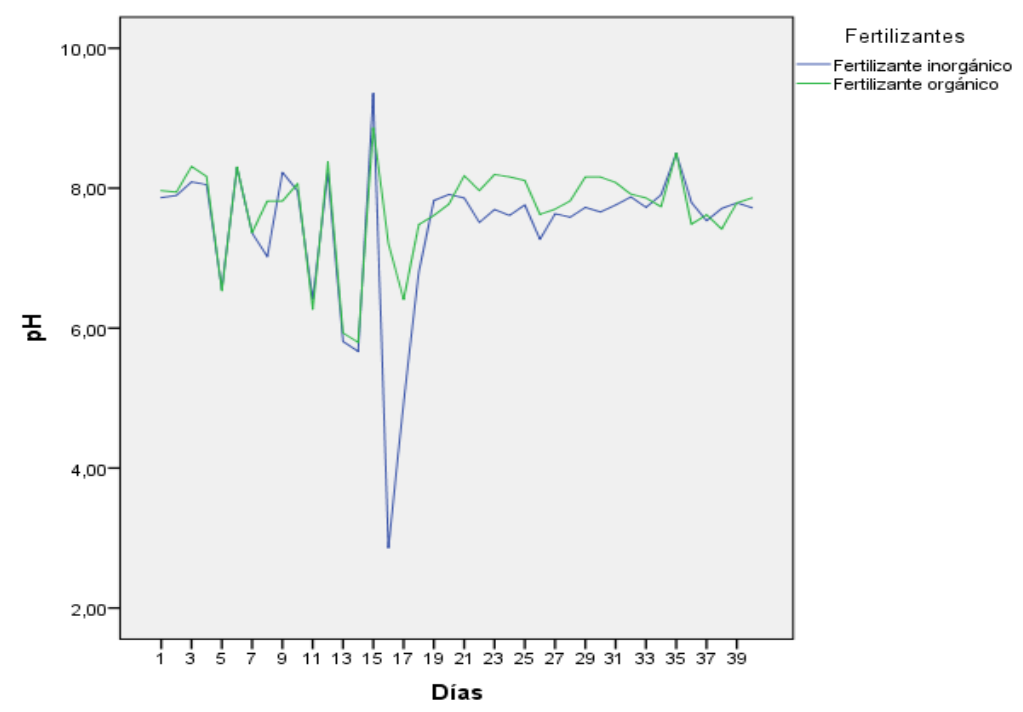

Figura No. 2: Comportamiento del pH en cultivo de camarón, utilizando fertilizante a base de Lombriabono versus fertilizante comercial.

Los niveles de temperatura durante el período de estudio para ambos tratamientos $\mathrm{T}_{1}$ (Fertilizante comercial) y $\mathrm{T}_{2}$ (Fertilizante Lombriabono), presentan una ligera tendencia a variar sus fluctuaciones en el tiempo, obteniendo datos mínimos de 28.3 ${ }^{\circ} \mathrm{C}$ para horas de la mañana y un máximo de $33.5^{\circ} \mathrm{C}$ para horas de la tarde en el $\mathrm{T} 1$, en el caso del $\mathrm{T} 2$ el valor mínimo de temperatura fue 28.2 para horas de la mañana y por la tarde para el mismo tratamiento tuvimos un máximo de $33.9^{\circ} \mathrm{C}$. Considerando lo dicho por Park et al. (2011), la temperatura óptima para la producción algal varía entre las especies, pero en general debe fluctuar entre $28^{\circ} \mathrm{C}$ y $35^{\circ} \mathrm{C}$. Si bien, en ambos tratamientos se manifestaron fluctuaciones muy marcadas, específicamente en las últimas dos semanas (mes de septiembre) del periodo del ensayo producto de los fenómenos tropicales, los cuales se presentaron con constantes lluvias y nubosidades, lo que disminuyó la temperatura del estanque, esta variable no influyo de manera significativa ya que los valores obtenidos no estaban fuera de los rangos óptimos de crecimiento para microalgas. 


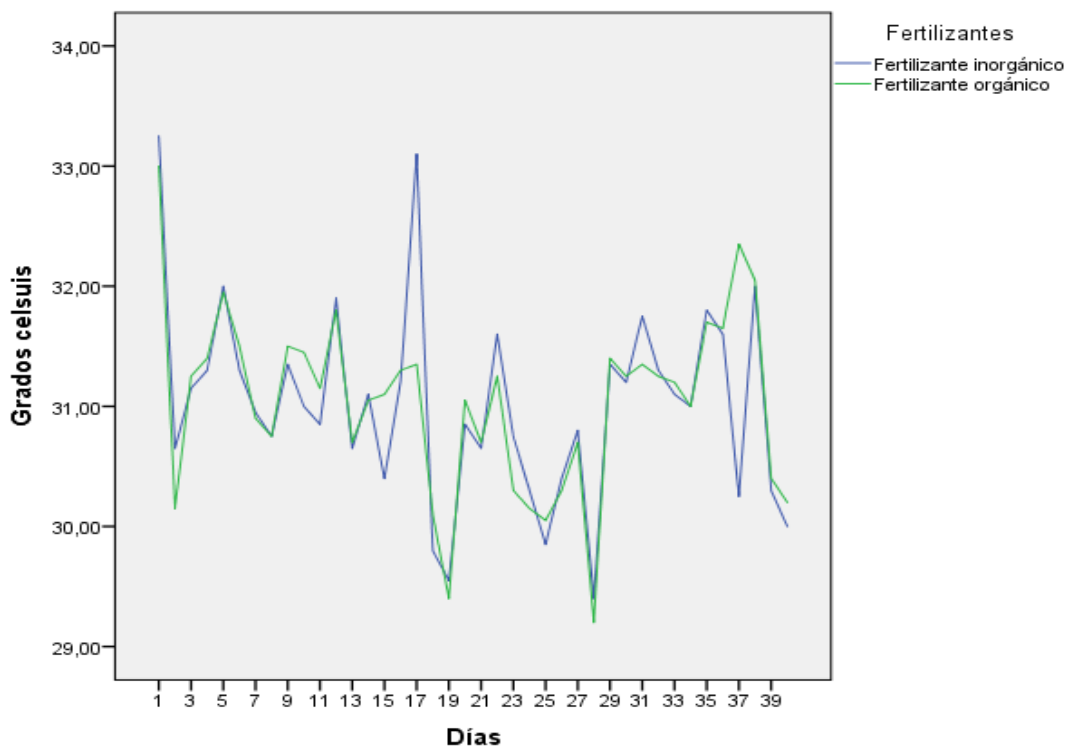

Figura No. 3: Comportamiento de la Temperatura en cultivo de camarón, utilizando fertilizante a base de Lombriabono versus fertilizante comercial.

Las concentraciones salinas disueltas durante el periodo de estudio para ambos tratamientos $\mathrm{T}_{1}$ (Fertilizante comercial) y $\mathrm{T}_{2}$ (Fertilizante Lombriabono), presentan una leve tendencia a variar sus fluctuaciones en el tiempo, obteniendo datos mínimos de $26 \%$ para horas de la mañana y un máximo de $33 \%$ para horas de la tarde en el T1, en el caso del T2 el valor mínimo de salinidad fue $27 \%$ o para horas de la mañana y por la tarde para el mismo tratamiento tuvimos un máximo de $33 \%$. Según Torzillo y Vonshak, (2013) citado por García-Romeral et al, (2016) el factor salinidad, afecta el crecimiento de las microalgas en función de la actividad osmótica, disminuye los procesos metabólicos y la replicación celular, cotejando con nuestros resultados, estos indican que los valores de la concentración salina presentan una tendencia próspera a medida que avanzaba en el tiempo. Sin embargo se perciben puntos muy marcados de salinidad influenciados por la época de invierno, en la cual la incidencia solar se prolongaba durante todo el día y la precipitación pluvial por las noches, creando diluciones entre el agua de la lluvia y el agua del estanque por las noches, recuperando sus concentraciones durante el día por efecto de la evaporación, asimismo, estas diluciones cede a que las aguas se mezclen produciéndose una redistribución de nutrientes en todo el estanque sin alterar la síntesis y actividad de la clorofila y desarrollo del fitoplancton. 


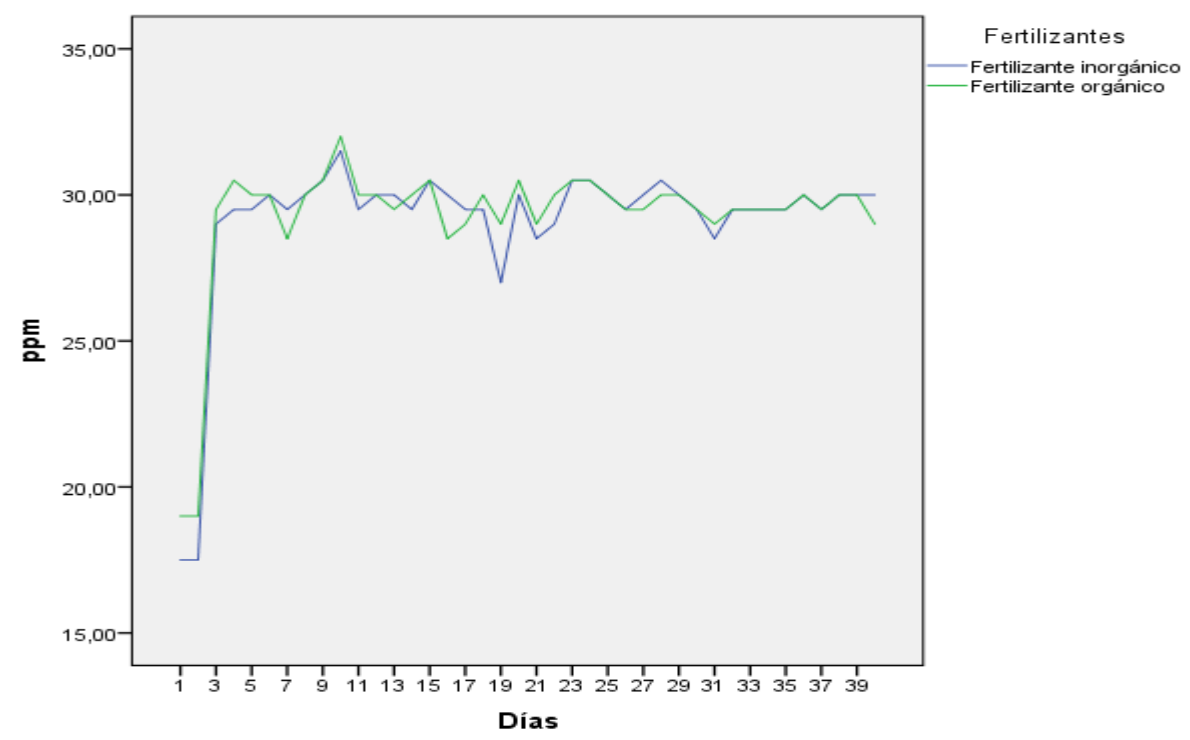

Figura No. 4: Comportamiento de la Salinidad en cultivo de camarón, utilizando fertilizante a base de Lombriabono versus fertilizante comercial.

Los niveles de turbidez durante el periodo de estudio para ambos tratamientos $\mathrm{T}_{1}$ (Fertilizante comercial) y $\mathrm{T}_{2}$ (lombriabono), presentan una tendencia a variar sus fluctuaciones en el tiempo, obteniendo datos mínimos de o $\mathrm{cm}$ y un máximo de 35 $\mathrm{cm}$ en la $\mathrm{T} 1$, en el caso del T2 el valor mínimo fue $60 \mathrm{~cm}$ y un máximo de $33 \mathrm{~cm}$ en cuanto a la lectura del disco sechi. En cuanto al comportamiento de la turbidez, en el cuerpo de agua se encontraron variaciones a lo largo del estudio donde los primeros nueve días obtuvimos una lectura de o cm con disco de Secchi, esto se debió a que el cuerpo de agua presentaban transparencia, a pesar de presentar nutrientes disponibles para el florecimiento algal por la fertilización realizada, pero debido a la influencia de valores altos del $\mathrm{pH}$ contenido en el agua de reservorio proveniente del océano afecto considerablemente el crecimiento de las microalgas en los primeros días del ensayo, posteriormente al disminuir el $\mathrm{pH}$ contenido en el agua proveniente del océano sumado los nutrientes disponibles en el cuerpo de agua producto de la fertilización se favoreció el crecimiento del fitoplancton. 


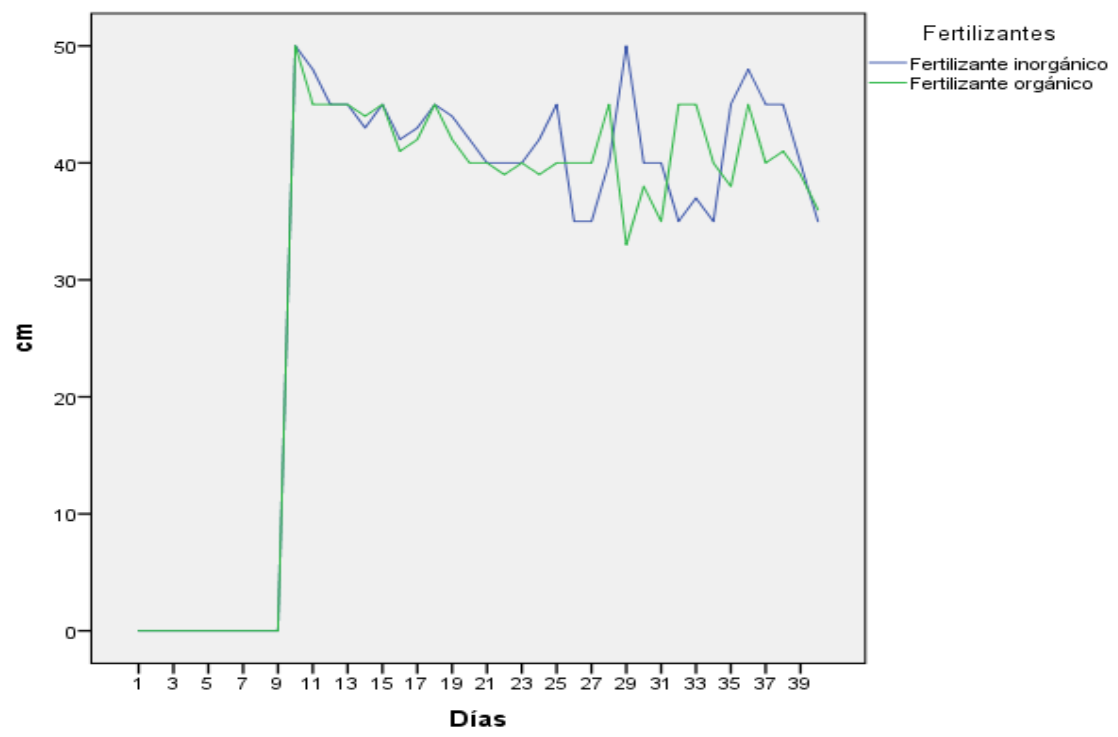

FiguraNo. 5: Comportamiento de la Turbidez en cultivo de camarón, utilizando fertilizante a base de Lombriabono versus fertilizante comercial.

La cantidad necesaria para la fertilización de un estanque siempre ha sido una polémica, puesto que este varía según el contenido del fertilizante, en el estudio se aprecia que al fertilizar ambos tratamientos a razón de 10olb/Ha la población de microalgas supera las $300,000 \mathrm{cel} . / \mathrm{ml}$, encontrando a los grupos de microalgas de mayor predominación el grupo de las diatomeas con 465,000 cel./ml seguido del grupo de las Chlorophytas con 167,500 cel./ml y por último pero no menos importante se encontró presencia de Cianophytas con 20,000 cel./ml esto debido a que el estudio se realizó en periodo de invierno, estos datos corresponden a la primera semana con el fertilizante inorgánico, para las siguientes 4 semanas el grupo de mayor predominancia fueron las Chlorophytas pero en ningún momento la densidad poblacional de microalgas disminuyo de 300,000 cel./ml con dicho fertilizante, con la diferencia que en la semana 5 hubo presencia de protozoos con 5,00o cel./ml. La predominancia de las Chlorophytas se debió principalmente a que el grupo de estas el 90\% es de hábitat de agua dulce y solo el 10\% es de hábitat marino a medida se avanzaba en el tiempo los periodos lluviosos eran más prolongados, propiciando diluciones en el cuerpo de agua situación que en el caso de este grupo resulta idóneo debido a su afinidad a los ambientes dulce acuícolas.

Por otro lado la densidad poblacional de microalgas con el fertilizante orgánico el grupo de mayor predominación fue las diatomeas con 270,000 cel./ml, seguido de las Chlorophytas 100,000 cel./ml, pero en este caso a diferencia del tratamiento anterior a pesar de estar en periodo de invierno no hubo presencia de Cianophytas, estos datos se corresponden a la primera semana, en las siguientes 4 semanas predominaron las 
Chlorophytas sobre el grupo de diatomeas teniendo densidades poblacionales por encima de $300,000 c e l . / \mathrm{ml}$, cabe mencionar que para las últimas dos semanas en este tratamientos se encontró presencia de Cianophytas, sin disminución de las 300,000 cel. $/ \mathrm{ml}$ de microalgas totales que deben estar presentes en un cuerpo de agua.

En base a los resultados obtenidos de la densidad poblacional de microalgas en ambos tratamientos y en contraste con lo dicho por Boyd y Daniels (1993), el rango total de microalgas fluctúa entre 300,000 a 400,000 cel./ml, por lo que la densidad poblacional en ambos tratamientos no estuvo en ningún momento por debajo de lo establecido por el autor.

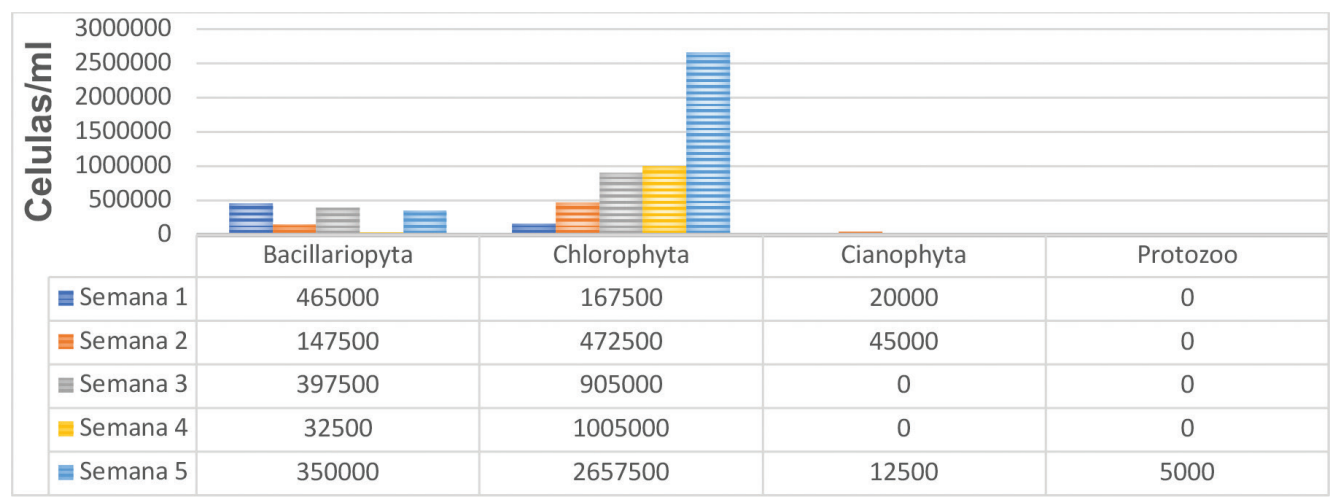

Figura No. 6: Grupos predominantes de microalgas con aplicación de fertilizante a base de Lombriabono.

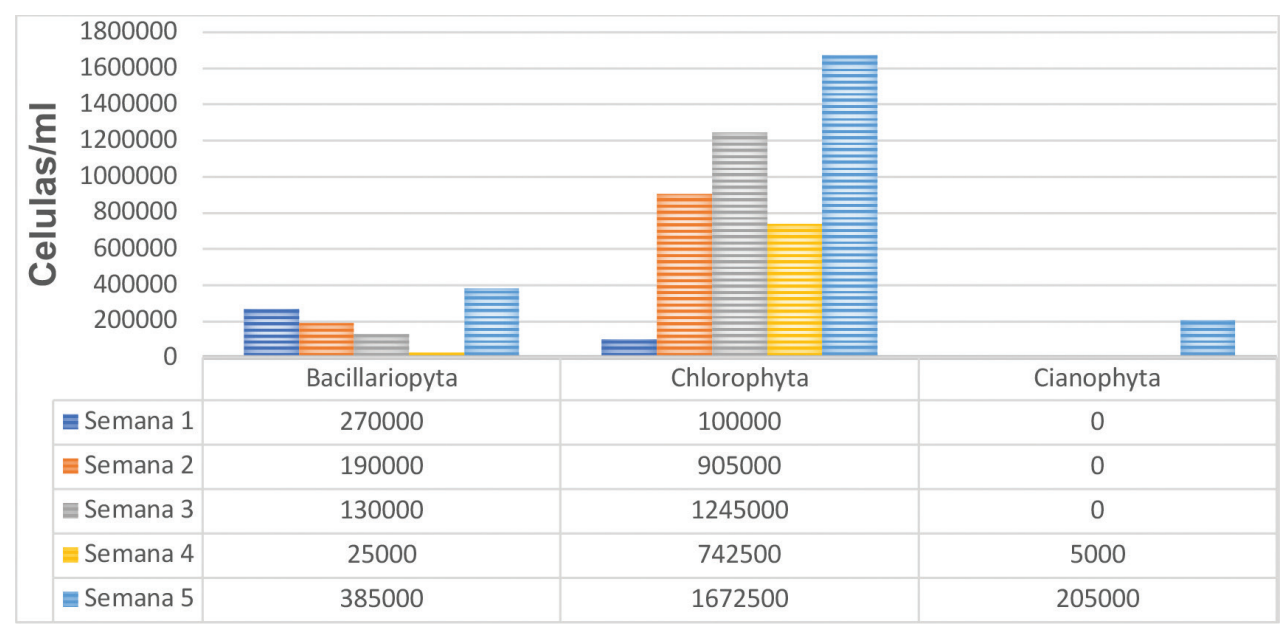

Figura No. 7: Grupos predominantes de microalgas con aplicación de fertilizante comercial. 
Con respecto a los índices de Shannon y de Simpson, el primero nos indica que es la cuantificación de la biodiversidad, es decir la heterogeneidad de una comunidad basándose en el número de las especies y su abundancia, aclarado esto al aplicar el índice de Shannon en el estudio nos indica que el fertilizante inorgánico presenta mayor diversidad de especie que el orgánico, dicha diferencia no es significativa, sin embargo ambos tratamientos no llegan a presentar el valor de 3 que requiere el índice para determinar que un cuerpo de agua presenta gran biodiversidad, esto se debió a que el estanque es un cuerpo de agua restringido donde no hay una recirculación constante de nutrientes y cabe destacar que en el estanque se encontraban organismos que se alimentan del fitoplancton por ende provoca una disminución en la diversidad fitoplanctónica.

Por otro lado, el índice de Simpson mide la abundancia de una o más especies dentro de una comunidad, dicho esto al aplicar el índice de Simpson nos indica que el fertilizante inorgánico y el orgánico presentaron una dominancia de la misma especie de microalgas con mayor relevancia de las especies de Cyclotella medunae, C. atomus, Navicula tripunctata, Nitzschia reversa, Cosmarium contractum, volvox aureus y Dunaliella tertiolecta para ambos tratamientos, esto gracias a que ambos proporcionaron a las microalgas nutrientes y condiciones óptimas como los parámetros físicos y químicos, que estos son los principales para su desarrollo y crecimiento.

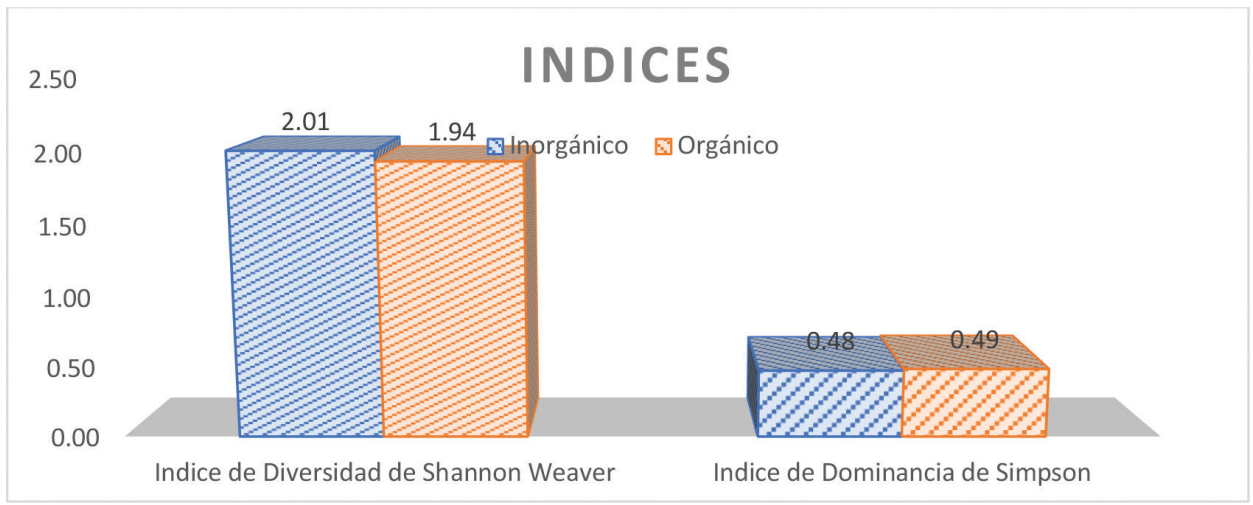

Figura No. 8: Contraste del Índices de diversidad de Shannon Weaver e Índice de Dominancia de Simpson

Las especies de microalgas expresadas en el siguiente cuadro son las que presentaron mayor frecuencia en los tratamientos, al mismo tiempo que son las especies que representan mayor predominancia y abundancia en ambos tratamientos. 
Tabla 1: Grupos y especies de microalgas de mayor frecuencia en el estudio.

\begin{tabular}{|c|c|c|}
\hline Tratamiento & Grupo & Especie \\
\hline \multirow{7}{*}{$\mathrm{T}_{1}$} & \multirow{4}{*}{ Bacillariophyta } & Cyclotella meduanae \\
\hline & & Cyclotella atomus \\
\hline & & Navicula tripunctata \\
\hline & & Nitzschia reversa \\
\hline & \multirow{3}{*}{ Chlorophyta } & Cosmarium Contractum \\
\hline & & Volvox aureus \\
\hline & & Dunaliella tertiolecta \\
\hline \multirow{7}{*}{$T_{2}$} & \multirow{4}{*}{ Bacillariophyta } & Cyclotella meduanae \\
\hline & & Cyclotella atomus \\
\hline & & Navicula tripunctata \\
\hline & & Nitzschia reversa \\
\hline & \multirow{3}{*}{ Chlorophyta } & Cosmarium Contractum \\
\hline & & Volvox aureus \\
\hline & & Dunaliella tertiolecta \\
\hline
\end{tabular}

\section{Conclusiones}

Los parámetros físicos y químicos (oxígeno, $\mathrm{pH}$, temperatura, salinidad y turbidez) monitoreados para ambos tratamientos (fertilizante comercial y lombriabono) no muestran variaciones relevantes durante el estudio, sin embargos los datos señalan que fenómenos como las lluvias y la evaporación provocaron alteraciones mínimas sin perjudicar el crecimiento microalgal.

Los datos establecen que en ambas condiciones experimentales al ser fertilizadas a razón de $100 \mathrm{Lb} / \mathrm{ha}$, no difieren en cuanto a calidad y cantidad de las comunidades fitoplanctónicas, ya que en ambos se superó las 300,000 cel./mL obteniendo mayor predominancia en Chlorophytas y diatomeas, presentando buena calidad del agua requeridas para el cultivo de Litopenaeus vannamei.

En cuanto a los índices de dominancia y diversidad Shannon-Weaver y Simpson respectivamente, ambos tratamientos resultaron con la misma cantidad de especies microalgales; su abundancia y diversidad presentan una leve discrepancia entre sí, sin embargo, ambos tratamientos no llegan a presentar el valor de 3 que requiere 
el índice para determinar que un cuerpo de agua presenta variedad en cuanto a su biodiversidad, esto debido a que un estanque acuícola es un ambiente restringido, diferente al medio natural.

Los grupos que se presentaron con mayor frecuencia durante el estudio en ambos tratamientos fueron las Bacillariophyta y Chlorophyta con las especie de Cyclotella meduanae, Cyclotella atomus, Navicula tripunctata, Nitzschia reversa, Cosmarium Contractum, Volvox aureus y Dunaliella tertiolecta, por otro lado la población de Cianophyta fue considerablemente mínima, ya que estas se presentan cuando existe una pobre calidad de agua por considerarse toxicas y no se presentaron en el estudio Dinoflagelados.

La aplicación de los dos tipos de fertilizantes orgánicos e inorgánicos no presentan diferencia significativa en el efecto promotor de la productividad primaria en un cultivo acuícola por lo tanto se acepta la hipótesis nula. El uso de fertilizantes orgánicos, en este caso el humus de lombriz, mantuvo la calidad de agua dentro de los intervalos óptimos para el estímulo del crecimiento y desarrollo de la productividad primaria al igual que el Fertilizante Inorgánico o químico. Lo cual nos indica que este tipo de fertilizante orgánico puede ser adecuado en el uso de la actividad camaronicola, beneficiando en la reducción de gastos en fertilizantes y alimento los cuales superan más del 50\% de los costos operacionales en esta actividad.

\section{Agradecimiento}

Esta publicación obtuvo el financiamiento de: El Fondo de Asistencia Internacional de los Estudiantes y Académicos Noruegos, (SAIH).

\section{Lista de referencias}

Benavente, J; Montañez, J; Aguilar, C; Méndez, A y Valdivia, B. (2012). Tecnología de cultivo de microalgas en fotobiorreactores. Divulgación científica, (1), 1.

Boyd, C y Daniels, H. (1993). Liming y fertization of Brackish water shrimp ponds.

Bhusan, C. y Yadav, B. (2003). Vermiculture for sustaintable agriculture. Indian farming digest. pp 11- 13 .

FAO (Organización de las naciones unidas para la alimentación y la agricultura). (2016). El Estado Mundial De La Pesca Y La Acuicultura 2016. Disponible en: http://www.fao.org/3/a-i5798s.pdf

García-Romeral J., Pavía-Gómez M., García T., Chirivela-Martorell J. y Serrano-Aroca A. (2016). Principios de Biotecnología y Bioingeniería en el cultivo de microalgas: importancia, problemas tecnológicos, tipos y sistemas de cultivo, crecimiento, 
factores limitantes, selección, aislamiento, esaclado y caracterización boquímica. NEREIS. Revista Iberoamericana Interdiciplinar de Métodos, Modelización y Simulación. Volumen 9. Universidad Católica de Valencia San Vicente Mártir, Valencia, España. ISSN 1888-8550. Pág. 115-129. Disponible en:

https://www.researchgate.net/publication/318946951_Principios_de_ Biotecnologia_y_Bioingenieria_en_el_cultivo_de_microalgas_importancia_ problemas_tecnologicos_tipos_y_sistemas_de_cultivos_crecimiento_factores_limitantes_seleccion_aislamiento_escalado_y

Guerra M. (2011). Humus de Lombriz Eisenia foetida para cultivar dos microalgas marinas como alimento para larvas de camarón. Tesis presentada en opción al Título Académico de Master en Biología con mención en Acuicultura. Centro de Investigaciones marinas. Universidad de La Habana. La Habana, Cuba.

Park, J., Craggs, R \& Shilton, A. (2011). Wastewater treatment high rate algal ponds for biofuel production. Bioresource Technology 102: 35-42.

Ruiz, A. (2011). Puesta en marcha de un cultivo de microalgas para la eliminación de nutrientes de un agua residual urbana previamente tratada anaeróbicamente. Universidad Politécnica De Valencia, España.

Ruiz, L. (2013). Uso del humus de lombriz como promotor del fitoplancton en cultivos intensivos experimentales a baja salinidad de camarón Litopenaeus vannamei. Instituto Politécnico Nacional, Sinaloa; México. 